10. Xiao CT, Giménez-Lirola LG, Gerber PF, Jiang YH, Halbur PG, Opriessnig T. Identification and characterization of novel porcine astroviruses (PAstVs) with high prevalence and frequent co-infection of individual pigs with multiple PAstV types. J Gen Virol. 2013;94:570-82. http://dx.doi.org/10.1099/ vir.0.048744-0

Address for correspondence: Bailey Arruda, 1850 Christensen Dr, Department of Veterinary Diagnostic and Production Animal Medicine, Iowa State University, Ames, IA 50011, USA; email: wilberts@iastate.edu

\section{Avian Influenza A(H7N9) Viruses Co-circulating among Chickens, Southern China}

\author{
Nianchen Wang, ${ }^{1}$ Minhua Sun, ${ }^{1}$ Wenqing Wang, ${ }^{1}$ \\ Guowen Ouyang, Zuxian Chen, You Zhang, \\ Bingbing Zhao, Siyu Wu, Jianni Huang, \\ Hailiang Sun, Ming Liao, Peirong Jiao
}

Author affiliations: South China Agricultural University, Guangzhou, China (N. Wang, W. Wang, G. Ouyang, Z. Chen, Y. Zhang, B. Zhao, S. Wu, J. Huang, H. Sun, M. Liao, P. Jiao); Institute of Animal Health, Guangdong Academy of Agricultural Sciences, Guangdong, China (M. Sun)

DOI: https://doi.org/10.3201/eid2312.170782

During 2016-2017, three avian influenza A(H7N9) viruses were isolated from chickens in southern China. Each virus had different insertion points in the cleavage site of the hemagglutinin protein compared to the first identified H7N9 virus. We determined that these viruses were double or triple reassortant viruses.

Since its first documentation on March 30, 2013, through Narch 16, 2017, avian influenza A(H7N9) virus has caused 5 epidemic waves of infection among humans in China, resulting in 1,307 laboratory-confirmed clinical cases and 489 deaths (1). According to reports of H7N9 virus outbreaks among humans in China, the virus clustered into the Yangtze River Delta lineage and the Pearl River Delta lineage (2). As with most low-pathogenicity avian influenza viruses, the early H7N9 avian influenza virus produced mild symptoms in domestic poultry and was therefore generally only detected through active virologic surveillance $(3,4)$.

${ }^{1}$ These authors contributed equally to this article.
In April 2017, H7N9 viruses (isolates A/chicken/ Guangdong/Q1/2016, A/chicken/Guangdong/Q26/2017, and A/chicken/Guangdong/Q39/2017, hereafter Q1, Q26, and Q39) were identified from lung samples of chickens that were collected from Guangdong, China, in June 2016 and January 2017. We sequenced all 8 genes of these viruses to trace the origin and clarify the genetic properties. The nucleotide sequences are available from GenBank (accession nos. MF280181-204).

The $\mathrm{H} 7$ hemagglutinin (HA) gene of all 3 viruses belonged to the Yangtze River Delta lineage A (Figure). However, unlike the early H7N9 virus, the HA genes were 1,695 bp and coded $565 \mathrm{aa}$, and the isolates had 4 inserted amino acids at cleavage sites ( KRTAR $\left.^{-} \mathrm{G}\right)$. In addition, Q26 and Q39 had 4 continuous basic amino acids at cleavage sites ( KRKRTAR $^{-} \mathrm{G}$ ), which is a characteristic of highly pathogenic avian influenza virus (Online Technical Appendix, Table 1, https://wwwnc.cdc.gov/EID/article/23/12/17-0782Techapp1.pdf). Q1 had a mutation (Q226L) at the receptor binding site of the HA protein, indicating a higher binding affinity for sialic acid $\alpha 2,6$, a characteristic of human cellsurface receptors (5).

Both Q1 and Q39 had an NA gene of Yangtze River Delta lineage A, whereas the NA gene of Q26 was of Pearl River Delta lineage (online Technical Appendix Table 1, Figure). A246T and R292K, which are related to drug resistance, had no substitution in the NA protein of the viruses we analyzed.

The polymerase basic (PB) 1 and 2, polymerase acidic, and nonstructural genes of Q1 and Q39 were all of Yangtze River Delta lineage A, and nucleoprotein genes were of Yangtze River Delta lineage B. The PB2 and nucleoprotein genes of Q26 were of Yangtze River Delta lineage A; PB1, polymerase acidic, and nonstructural genes of Q26 were clustered to the Pearl River Delta lineage (online Technical Appendix Table 1, Figure). E627K and D701N had no substitution in the PB2 protein of the viruses, which was thought to contribute to the adaptation, replication, and virulence of influenza viruses in humans and mice $(6,7)$.

Of particular note, the matrix $\mathrm{M}$ gene of Q1 clustered into A/goose/Guangdong/1/96-lineage (H5N1) (GSGD96 lineage) and had a nucleotide of $94.8 \%$. However, the matrix genes of Q26 and Q39 clustered into Yangtze River Delta lineage B of H7N9 virus (online Technical Appendix Table 1, Figure).

To clarify the pathogenicity and transmission of the virus, we inoculated 11 chickens with each isolate $\left(10^{6} 50 \%\right.$ egg infectious dose $\left[\mathrm{EID}_{50}\right]$ in $0.1 \mathrm{~mL}$ of phosphate-buffered saline) and 3 chickens with $0.1 \mathrm{~mL}$ phosphate-buffered saline as the control group. We observed all chickens for clinical symptoms for 14 days. The infected chickens exhibited anorexia and signs of depression at 2 days postinoculation (DPI). The Q1 inoculated group died within 4 DPI, Q26 within 3 DPI, and Q39 within 2 DPI; contact 
groups of Q1, Q26, and Q39 died within 7 DPI, 4 DPI, and 5 DPI, respectively. These viruses replicated systemically in chickens of inoculated groups and were detected in all of the tested organs by 3 DPI. The mean virus titers in the trachea, liver, spleen, kidney, brain, and lung were 4.92 $8.42 \log _{10}$ EID $_{50} / \mathrm{g} / 0.1 \mathrm{~mL}$ (online Technical Appendix Table 2). The Q1, Q26, and Q39 virus titers in lung samples for each contact group were 7.5-8.1 $\log _{10} \mathrm{EID}_{50} / \mathrm{g} / 0.1 \mathrm{~mL}$.

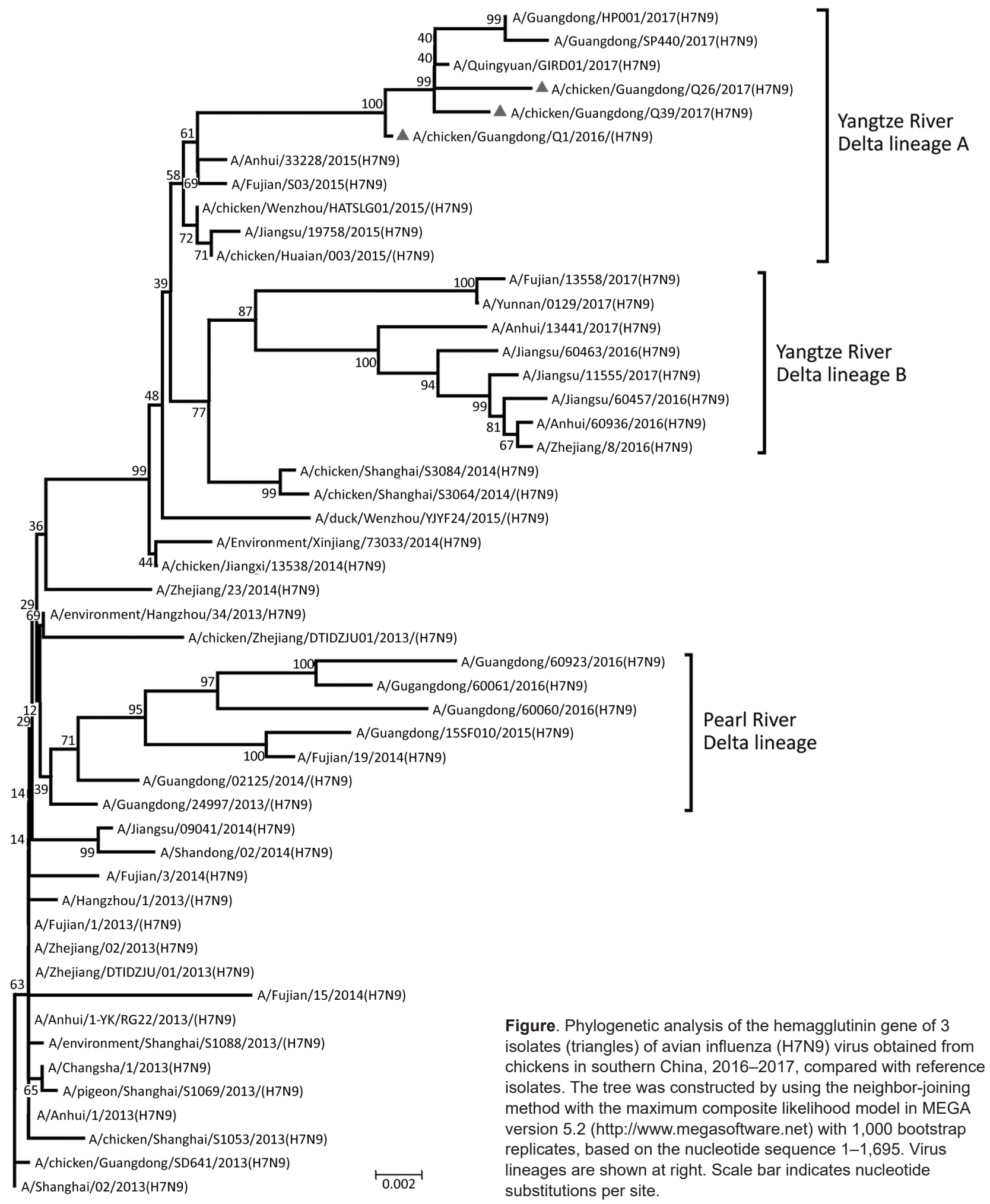


Therefore, the new H7N9 viruses were highly pathogenic to chickens when compared with the early H7N9 virus and could transmit among chickens by contact.

The biological features of H7N9 virus and its pandemic potential have caused global concern (8). The early H7N9 viruses lacked the basic HA cleavage site, exhibited low pathogenicity, and caused mild or no disease in poultry (9). The cleavage site in HA protein of the isolates we analyzed were KGKRTAR $^{-} \mathrm{G}$ or $\mathrm{KRKRTAR}^{-} \mathrm{G}$. They had high pathogenicity and replication in chickens and could transmit among chickens by contact. Therefore, these new H7N9 viruses could cause a pandemic among poultry and humans in China.

Molecular evolution showed that Q1 was a triple reassortant virus (H5, H7, and H9 subtypes) consisting of Yangtze River Delta A and B lineages of H7N9 and GSGD96 lineage of H5N1. The Q26 and Q39 viruses were both double reassortant avian influenza viruses (H7 and $\mathrm{H} 9$ subtype), as was the early H7N9 virus (Figure; online Technical Appendix Table 1, Figure). Therefore, the $3 \mathrm{H} 7 \mathrm{~N} 9$ viruses we isolated have 2 kinds of insertions in the cleavage sites and were likely derived from different lineages of H7N9 viruses, or even from different subtypes that were co-circulating in southern China during 2016-2017.

\section{Acknowledgments}

We thank the Key Laboratory of Zoonosis, Ministry of Agriculture; the National and Regional Joint Engineering Laboratory for Medicament of Zoonosis Prevention and Control; the Key Laboratory of Animal Vaccine Development, Ministry of Agriculture; and the Key Laboratory of Zoonoses Control and Prevention of Guangdong.

This work was supported by grants from the National Key Research and Development Program of China

(2016YFD0500207), the National Natural Science Foundation of China (U1501212), the Natural Science Foundation of Guangdong Province (2016A030308001), and Basic Research (Discipline Layout) of Shenzhen (JCYJ20160323163102764).

Mr. Nianchen Wang is a student in the Master of Science program in the College of Veterinary Medicine, South China Agricultural University. His primary research interest is the epidemiology and pathogenesis of avian influenza viruses.

\section{References}

1. World Health Organization. Update on monthly risk assessment summary [cited 2017 Apr 20]. http://www.who.int/influenza/ human_animal_interface/Influenza_Summary_IRA_HA_ interface $04 \quad 20$ 2017.pdf?ua $=1$

2. Wang D, Yang L, Zhu W, Zhang Y, Zou S, Bo H, et al. Two outbreak sources of influenza A (H7N9) viruses have been established in China. J Virol. 2016;90:5561-73. http://dx.doi.org/ 10.1128/JVI.03173-15

3. Kang M, He J, Song T, Rutherford S, Wu J, Lin J, et al. Environmental sampling for avian influenza A(H7N9) in live-poultry markets in Guangdong, China. PLoS One. 2015; 10:e126335. http://dx.doi.org/10.1371/journal.pone.0126335

4. Shi J, Deng G, Liu P, Zhou J, Guan L, Li W, et al. Isolation and characterization of $\mathrm{H} 7 \mathrm{~N} 9$ viruses from live poultry marketsimplication of the source of current H7N9 infection in humans. Chin Sci Bull. 2013;58:1857-63. https://doi.org/10.1007/ s11434-013-5873-4

5. Ramos I, Krammer F, Hai R, Aguilera D, Bernal-Rubio D, Steel J, et al. H7N9 influenza viruses interact preferentially with $\alpha 2,3-$ linked sialic acids and bind weakly to $\alpha 2,6$-linked sialic acids. J Gen Virol. 2013;94:2417-23. http://dx.doi.org/10.1099/vir.0.056184-0

6. Li Z, Chen H, Jiao P, Deng G, Tian G, Li Y, et al. Molecular basis of replication of duck H5N1 influenza viruses in a mammalian mouse model. J Virol. 2005;79:12058-64. http://dx.doi.org/10.1128/JVI.79.18.12058-12064.2005

7. Hatta M, Gao P, Halfmann P, Kawaoka Y. Molecular basis for high virulence of Hong Kong H5N1 influenza A viruses. Science. 2001;293:1840-2. http://dx.doi.org/10.1126/science.1062882

8. Zhou J, Wang D, Gao R, Zhao B, Song J, Qi X, et al. Biological features of novel avian influenza A (H7N9) virus. Nature. 2013;499:500-3. http://dx.doi.org/10.1038/nature12379

9. World Health Organization. Update on analysis of recent scientific information on avian influenza A (H7N9) virus [cited 2017 Feb 10]. http://www.who.int/influenza/human_animal_interface/avian_ influenza/riskassessment_AH7N9_201702/en/

Address for correspondence: Peirong Jiao or Ming Liao, College of Veterinary Medicine, South China Agricultural University, 483 Wushan Rd, Guangzhou 510642, China; email: prjiao@scau.edu.cn ormliao@scau.edu.cn

\section{Rabies and Distemper Outbreaks in Smallest Ethiopian Wolf Population}

\author{
Jorgelina Marino, Claudio Sillero-Zubiri, \\ Asefa Deressa, Eric Bedin, Alemayehu Bitewa, \\ Fekadu Lema, Gebeyehu Rskay, Ashley Banyard, \\ Anthony R. Fooks
}

Author affiliations: University of Oxford, Oxford, UK (J. Marino, C. Sillero-Zubiri, E. Bedin); International Union for Conservation of Nature Species Survival Commission Canid Specialist Group, Oxford (C. Sillero-Zubiri); Ethiopian Public Health Institute, Addis Ababa, Ethiopia (A. Deressa); Environmental, Forest, Wildlife Development and Protection Authority, Bahir Dar, Ethiopia (A. Bitewa); Ethiopian Wolf Conservation Programme, Bale Robe, Ethiopia (J. Marino); Ethiopian Wolf Conservation Programme, Bahir Dar (F. Lema, G. Rskay); Animal and Plant Health Agency, Weybridge, UK (A. Banyard, A.R. Fooks)

DOI: https://doi.org/10.3201/eid2312.170893 